\title{
Raucherentwöhnung: Ein Thema für jeden Arzt
}

- Die Sinnhaftigkeit des Ausstiegs aus der Tabakabhängigkeit steht außer Zweifel und die Chance zur effektiven Prävention sollte auch in der Facharztpraxis nicht ungenutzt bleiben.

Aber wollen die Patienten überhaupt darauf angesprochen werden und würden sie einem ärztlich geleiteten Ausstieg zustimmen? Für die Mehrzahl der Raucher trifft dies tatsächlich zu. Ein neues Raucherentwöhnungsprogramm mit Bestandteilen, die ihre Effektivität bereits bewiesen haben, macht es dem Arzt besonders leicht, sich aktiv für den Rauchstopp seiner Patienten zu engagieren, weil es mit geringem Zeitaufwand umzusetzen ist. Es beginnt mit drei einfachen Fragen, die den Patienten nicht in die Enge treiben, und die somit keinen Widerstand hervorrufen: Rauchen Sie? Wie denken Sie über das Rauchen? Wären Sie gerne rauchfrei? Im Anschluss wird ein fester Tag für das Aufhören vereinbart. Vorher findet ein Termin statt mit Beratung zum Ablauf der Entwöhnung, Erhebung der Raucheranamnese etc.

Ab dem Tag des Rauchstopps erhält der Patienten eine Nikotinersatz-Therapie, z.B. mit NICORETTE ${ }^{\circledast}$ TX Pflaster. Während der Entwöhnungsphase kontaktiert man den Patienten am dritten Tag sowie nach 14 und 28 Tagen telefonisch und erkundigt sich, wie es ihm ergangen ist und ob er rauchfrei ist. Am Ende der Stabilisierungsphase nach 12 Wochen ist noch ein Abschlussgespräch vorgesehen, um die Motivation zu festigen und zum Umgang mit Rückfällen zu beraten. Das Engagement lohnt sich auch für den Arzt: Ein Patient, der es mit ärztlicher Hilfe geschafft hat, vom Rauchen loszukommen, wird ein dankbarer und treuer Patient sein! Das Programm kann unter www.

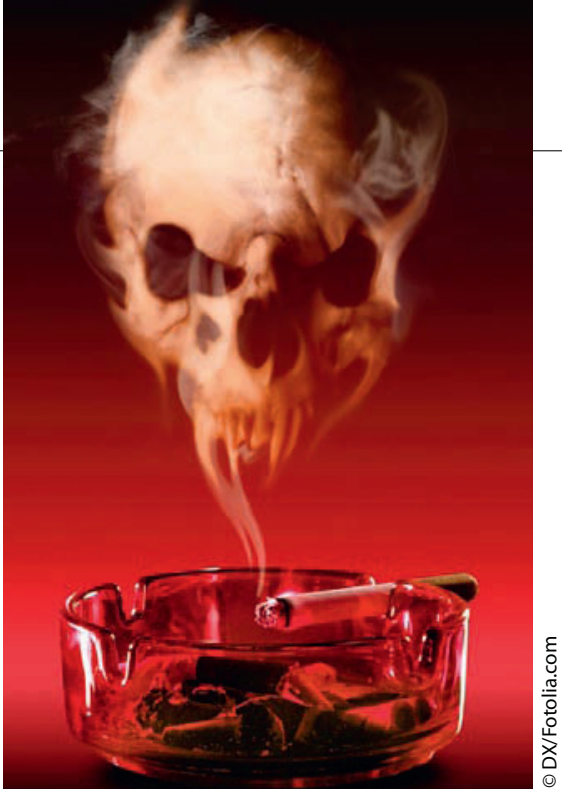

einfach-erfolgreich-rauchfrei.de oder per Fax (09134/7073214) unter dem Stichwort „EINFACH ERFOLGREICH RAUCHFREI" angefordert werden. Es ist kostenlos als Printversion oder CD-Rom erhältlich.

$A B$

Pressekonferenz „Rauchen: Entwöhnungstherapie - von Ärzten für Ärzte", Bad Orb, 27. Oktober 2010, Veranstalter: Johnson \& Johnson

\section{Bronchitis-Therapie: Mukoziliäre Clearance wieder herstellen}

— Bei Atemwegsinfekten wie der Bronchitis ist die mukoziliäre Clearance gestört, die entzündete Bronchialschleimhaut bildet vermehrt zähes Sekret, die Bewegungsfähigkeit der Zilien ist deutlich eingeschränkt. Für eine rasche Linderung der Beschwerden und für die Wiederherstellung der natürlichen Schutzmechanismen ist die Normalisierung der Schleimschutzschicht vorrangig. Hier kann sich der Einsatz von Thymian- und Efeuextrakten wie sie im Phytopharmakon Bronchi- pret $^{\oplus}$ enthalten sind, als hilfreich erweisen. Die Inhaltsstoffe - Thymian und Efeuextrakt im Saft und Thymian- und Primelwurzeltrockenextrakt in der Tablette - wirken sekretolytisch, antiphlogistisch und bronchospasmolytisch. Der Einsatz synthetischer Sekretolytika sollte dagegen laut Dr. Riedl-Seifert, Kassel, nicht unkritisch erfolgen: „Die meisten Schleimlöser zerstören die Integrität des bronchialen Mukus und führen zur Verflüssigung. Doch das verflüssigte Sekret lässt sich schlecht ab- husten, da der ursprünglich zusammenhängende Sekretfilm reißt", so Riedel-Seifert. Anders die Pflanzenextrakte in Bronchipret ${ }^{\oplus}$ : Sie regulieren die Überproduktion von zähem festsitzendem Schleim und normalisieren das Sekret. Durch die sekretolytische, antiphlogistische und bronchospasmolytische Wirung verschaffen sie rasche Linderung mit nachhaltigem Effekt.

gz

Nach Informationen von Bionorica

\section{Erkältung: Antibakterielle Wirkung mit großer Reichweite}

— Erkältungen sind definiert als virale Infektion der Schleimhäute der oberen Atemwege. Wo jedoch die Infektion ihren Ausgang nimmt, variiert von Fall zu Fall ebenso wie die Lokalisation und Ausprägung der Symptome. Vor diesem Hintergrund weisen Experten wie Prof. Dr. med. Ralph Mösges, HNO-Arzt an der Universität Köln, darauf hin, dass die Reichweite fast aller Erkältungsmittel sehr begrenzt ist. So können Nasensprays nur bei Rhinitis bzw. Sinusitis Abhilfe schaffen, Lutschpastillen oder Tees nur bei Beschwerden in Mundhöhle oder Rachen, nicht aber im Larynx. Das Erkältungs- spray Locabiosol ${ }^{\circledR} S$ vereint dagegen die Vorteile oraler und nasaler Applikationsformen und reicht beim Inhalieren noch weit in die Trachea hinein. Es wird mit zwei verschiedenen Sprühaufsätzen geliefert. Fein vernebelt als Aerosol gelangt es an alle kritischen Punkte der oberen Atemwege ohne ein unangenehmes Taubheitsgefühl hervorzurufen. Locabiosol ${ }^{\circledR S}$ wirkt antiphlogistisch und lokal antibakteriell. Diese doppelte Wirkung ist dem natürlichen Inhaltsstoff Fusafungin zu verdanken, der durch Fermentation aus der Pilzart Fusarium lateritium gewonnen wird.
Als Lokalantibiotikum, von dem auch nach 35 Jahren Anwendung keine Resistenzen oder Kreuzresistenzen bekannt sind, schont Fusafungin die natürliche Flora der Atemwegsschleimhaut und kann unter Umständen sogar dem Einsatz systemischer Antibiotika vorbeugen. Fusafungin bekämpft darüber hinaus den Pilz Candida albicans und vermag im Gegensatz zu den meisten systemischen Antibiotika Sekundärcandidosen zu verhindern.

\section{gz}

Nach Informationen von STADA Arzneimittel 